\title{
Comprehensive bioinformatic analysis identified m6A methylation as an important regulator in mediating immune response during idiopathic pulmonary arterial hypertension
}

$\mathrm{Na} \mathrm{Liu}$

Department of Cardiology/Cardiac Catheterization Lab, Second Xiangya Hospital, Central South University

Yunhong Zeng

Department of Cardiology, Hunan Children's Hospital

Ting Huang

Department of Cardiology, Hunan Children's Hospital

Wanyun Zuo

Department of Cardiovascular Medicine, The Second Xiangya Hospital of Central South University, Changsha, Hunan, China

\section{Yunbin Xiao}

Department of Cardiology, Hunan Children's Hospital, Changsha, Hunan, China

\section{Biao Li}

Department of Cardiovascular Medicine, The Second Xiangya Hospital of Central South University, Changsha, Hunan, China

\section{Zhenghui Xiao}

Department of Cardiology, Hunan Children's Hospital, Changsha, Hunan, China

\section{Yaozhong Liu}

Department of Cardiovascular Medicine, The Second Xiangya Hospital of Central South University, Changsha, Hunan, China

Qiming Liu ( $\square$ qimingliu@csu.edu.cn )

Second Xiangya Hospital

\section{Research}

Keywords: N6-methyladenosine (m6A) methylation, pulmonary arterial hypertension (PAH), weighted gene co-expression network analysis (WGCNA), immune response

Posted Date: September 18th, 2020 
DOI: https://doi.org/10.21203/rs.3.rs-64337/v1

License: (c) (1) This work is licensed under a Creative Commons Attribution 4.0 International License. Read Full License 


\section{Abstract}

\section{Background}

Despite its functional importance in various fundamental bioprocesses, studies of N6-methyladenosine $\left(\mathrm{m}^{6} \mathrm{~A}\right)$ in the pulmonary arterial hypertension (PAH) are lacking. Here we studied the potential relevance of $\mathrm{m}^{6} \mathrm{~A}$ RNA methylation and immune response in PAH development.

Methods

We constructed a monocrotaline (MCT) induced PAH rat model and performed Methylated RNA immunoprecipitation sequencing (MeRIP-Seq). The 18 idiopathic PAH (IPAH) microarray data obtained from the GEO database was used to construct co-expression networks by weighted gene co-expression network analysis (WGCNA). CIBERSORT was used to investigate the effect of $\mathrm{m}^{6} \mathrm{~A}$ methylation on immune cell infiltration during $\mathrm{PAH}$.

Results

A differential pattern of $\mathrm{m}^{6} \mathrm{~A}$ abundance, mainly up-methylation, was observed in the lung tissues of rats with MCT induced PAH. By WGCNA, multi-list pathway enrichment analysis and protein-protein interaction (PPI) analysis, we found that $\mathrm{m}^{6} \mathrm{~A}$ methylation modification may play important roles in mediating immune response during PAH. CYBERSORT algorithm indicated that the $\mathrm{m}^{6} \mathrm{~A}$ methylation can drive monocyte to form M1 macrophage, which may be mediated by CCR5 and CXCL9.

\section{Conclusion}

Collectively, $\mathrm{m}^{6} \mathrm{~A}$ landscape is altered in PAH. We summarize newly discovered $\mathrm{m}^{6} \mathrm{~A}$ in controlling immune response, which caused activation of $\mathrm{M} 1$ macrophage during PAH. It's provided a novel insight into the therapeutic mechanisms of PAH.

\section{Background}

Pulmonary arterial hypertension (PAH) is a condition characterized by increased pulmonary arterial pressure and vascular remodeling of the small pulmonary arteries. PAH develops either as an idiopathic condition or in association with various underlying diseases such as collagen vascular disease, portal hypertension, or HIV infection $(1,2)$. The incidence of PAH ranges from 2.0 to 7.6 cases per million adults per year, and its prevalence varies from 11 to 26 cases per million adults. The incidence of PAH is fourfold higher in women than in men, but survival is paradoxically worse in men with $\operatorname{PAH}(3,4)$. Despite striking progress in the development of diagnosis and therapeutics in the last three decades, PAH remains relatively incurable and the limited efficacy of current treatment options possibly results from an incomplete understanding of the pathophysiology of this cardiopulmonary disorder(5). 
Epigenetic modifications, such as DNA methylation, histone modifications, and noncoding RNAs, may play important roles in regulating the pathogenesis of $\mathrm{PAH}(1)$. As a bridge to pass the genetic information from DNA to proteins RNA, RNA is an important part of the central dogma, and its various chemical modifications are involved in the regulation of various biological processes(6). N6-methyladenosine $\left(m^{6} A\right)$ of RNA transcripts is the most prevalent modification found in many classes of $\operatorname{RNA}(7,8)$. The abundance of $\mathrm{m}^{6} \mathrm{~A}$ on RNA is determined by the dynamic interplay between its methyltransferases ("writers", such as METTL3-METTL14 complex(9)), and demethylases ("erasers", including FTO(10) and ALKBH5(11)). The binding proteins ("readers", such as YTH domain-containing proteins $(12,13)$ ) then mediate the effect of $\mathrm{m}^{6} \mathrm{~A}$ modifications on RNA processing or metabolisms, including alternative splicing $(14,15)$, export $(16,17)$, stability $(18,19)$, and translation $(20,21)$.

Recent studies have suggested the involvement of the immune response during PAH. Inflammatory cell infiltrations can be observed around the pulmonary artery, such as macrophages dendritic cells, mast cells, $T$ lymphocytes, and B lymphocytes(22). These immune cells play an important role in the vascular remodeling characteristic of PAH and might be important targets for PAH therapy. Nevertheless, how the immune system is activated and regulated remains poorly understood. $\mathrm{m}^{6} \mathrm{~A}$ RNA modifications might be a novel immunoregulatory factor(23).

Weighted gene co-expression network analysis (WGCNA) is a system biology algorithm for describing the correlation patterns among genes across samples(24). WGCNA can identify and cluster highly correlated genes into the same module. Furthermore, this method can also be used for relating modules to external clinical traits. By far, WGCNA has been validated as a valuable method to identify underlying mechanisms, potential biomarkers, or therapeutic targets in different types of diseases.

In our study, we first constructed a PAH rat model and conducted the $\mathrm{m}^{6} \mathrm{~A}$ methylation sequencing analysis. We then analyzed 18 idiopathic PAH (IPAH) patients microarray data from the GEO database to identify $\mathrm{m}^{6} \mathrm{~A}$ methylation correlated modules by using the method of WGCNA. Afterward, we applied the CIBERSORT algorithm to investigate the effect of $\mathrm{m}^{6} \mathrm{~A}$ methylation on immune cell infiltration during PAH. Findings from our study may contribute to novel regulators and therapeutic targets in PAH from an epigenomic perspective.

\section{Material And Methods}

\subsubsection{Establishment and verification of rat model of PAH induced by MCT}

The study was approved by the Institutional Animal Care and Use Committee of the Second Xiangya Hospital of Central South University and complied with the standards in the Guide for the Care and Use of Laboratory Animals. Sprague-Dawley rats (specific pathogen-free (SPF), male, 180-200 g, 6 weeks old, $\mathrm{n}$ = 5) were obtained from Changsha Tianqin Biotechnology Company (China). The rats were randomized to the control $(n=2)$ and PAH groups $(n=3)$. The rats in the PAH group were intraperitoneally injected with monocrotaline (MCT) $\left(60 \mathrm{mg} \cdot \mathrm{kg}^{-1}\right.$, Sigma, C2401), while the rats in the control group were injected intraperitoneally with the same volume of saline. All rats were housed on a $12 \mathrm{~h}$ light/dark cycle with free 
access to food and water. After 4 weeks of feeding, rats were anesthetized with $1 \%$ sodium pentobarbital (130 $\mathrm{mg} \cdot \mathrm{kg}^{-1}$ ) for echocardiography and right heart catheterization. Echocardiography was used to record the tricuspid regurgitation velocity, tricuspid annulus contraction rate, tricuspid annular plane systolic excursion (TAPSE) and pulmonary artery blood flow acceleration time (PAAT). After echocardiographic examination, We performed a right heart catheter to measure pulmonary artery pressure.

Rats were sacrificed by cervical dislocation after deep anesthesia. Then, heart tissues were removed and segregated. The ratio of right ventricle to left ventricle plus ventricular septum [RV/ $(L V+S)]$ was used as an index of right ventricular hypertrophy. Lung tissues were excised and immediately frozen at liquid nitrogen or fixed in $4 \%$ buffered paraformaldehyde solution.

\subsubsection{Histological analysis}

The lung tissues obtained in each group were placed in $4 \%$ buffered paraformaldehyde solution overnight, and then dehydrated and embedded in paraffin. Then, all lung tissues were sliced into $5 \mu \mathrm{m}-$ thick sections, fixed on a glass slide and baked dry. The staining procedures were performed according to the instructions. Briefly, the sections were soaked in xylene, ethanol in gradient concentration and hematoxylin, respectively, and sealed with resin. After dryness, pulmonary vascular morphology was observed and photographed under optical microscope. Last, the ratio of pulmonary small artery wall thickness and muscularization was calculated.

\subsubsection{RNA preparation}

For each group, four biological replicates were selected, of which every two were combined into one. Then, total RNA of tissue was extracted using TRIzol reagent (Invitrogen Corporation, CA, USA) following the manufacturer's instructions. The Ribo-Zero rRNA Removal Kit (Illumina, Inc., CA, USA) was used to reduce the ribosomal RNA content of total RNAs. Then, the RNA was chemically fragmented into fragments of about 100 nucleotides in length using fragmentation buffer (Illumina, Inc.).

2.1.4 Methylated RNA immunoprecipitation sequencing (MeRIP-Seq) and library construction MeRIP-Seq was performed following a previously reported procedure with slight modifications. Briefly, $\mathrm{m}^{6}$ A RNA immunoprecipitation was performed with the GenSeqTM $\mathrm{m}^{6}$ A RNA IP Kit (GenSeq Inc., China) by following the manufacturer's instructions. Both the input sample without immunoprecipitation and the $\mathrm{m}^{6} \mathrm{~A}$ IP samples were used for RNA-seq library generation with NEBNext ${ }^{\circledR}$ Ultra II Directional RNA Library Prep Kit (New England Biolabs, Inc., USA). The library quality was evaluated with BioAnalyzer 2100 system (Agilent Technologies, Inc., USA). Library sequencing was performed on an illumina Hiseq instrument with 150 bp paired-end reads.

\subsection{Microarray data collection and processing}

Materials of GSE15197 were downloaded from the NCBI Gene Expression Omnibus (GEO; https://www.ncbi.nlm.nih.gov/geo/) database. The dataset contains lung tissue transcriptomic profiles from 18 IPAH patients. The median expression values among all multiple probe IDs were selected to represent the corresponding gene symbol, leading to the identification of 18612 unique genes across 18 
samples. Human gene annotation file (GRCh38 release 99 gene transfer format, ensembl.org/index.html) was applied to annotate genes and the 15934 protein-coding genes were selected for further analysis.

\subsection{Estimation of $\mathrm{m}^{6} \mathrm{~A}$ methylation score and implementation of weight correlation network analysis (WGCNA)}

We constructed gene signatures of $\mathrm{m}^{6} \mathrm{~A}$ writer (METTL3, METTL14, METTL16, WTAP, KIAA1429, ZC3H13, RBM15/RBM15B), and $\mathrm{m}^{6} \mathrm{~A}$ eraser (ALKBH5, FTO) as suggested by Yang et.al (25). We then computed the Gene Set Variation Analysis (GSVA) enrichment score of $m^{6} A$ writer and $m^{6} A$ eraser across the 18 samples using the 'GSVA' package(26) in R software. The estimated $\mathrm{m}^{6} \mathrm{~A}$ methylation score was calculated by substrating $\mathrm{m}^{6} \mathrm{~A}$ eraser score from $\mathrm{m}^{6} \mathrm{~A}$ writer score. WGCNA was accomplished with the $\mathrm{R}$ package 'WGCNA'(24). According to the standard variation value of gene, we ranked them from largest to smallest and only selected the top 2000 as input for WGCNA. A power of $\beta$ value was introduced so that it could transform the similarity matrix into an adjacency matrix. In this study, $\beta=5$ was used as a soft threshold parameter to ensure a scale-free network. The Dynamic Tree Cut method was applied to generate modules with the following major parameters to avoid the generation of too many modules: deepSplit of 2, minModuleSize of 30, and the height cut-off was set as 0.25 (modules were merged if their similarity was $>0.75$ ). Module eigengenes (MEs) referred to the first principal component of all gene expression levels in the module, and therefore, it was reasonable to consider that MEs represented all genes within a specific module. According to Pearson's correlation tests, we further identified the association between MEs and $\mathrm{m}^{6} \mathrm{~A}$ methylation score. Within the most relavant module, those positively correlative with $\mathrm{m}^{6} \mathrm{~A}$ methylation score were made subjected to further analysis.

\subsection{Pathway enrichment analysis}

Metascape (https://Metascape.org/) is a web-based portal designed to provide a comprehensive gene list annotation and analysis resource for biologists(27). To gain insights into biological roles of $\mathrm{m}^{6} \mathrm{~A}$ methylation correlated genes identified from WGCNA and upmethylated genes identified from $\mathrm{m}^{6} \mathrm{~A}$-seq, we conducted pathway enrichment analysis in Metascape tools using Gene Ontology biological process (GO BP), Kyoto Encyclopedia of Genes and Genomes (KEGG), and Reactome(27). By inputting the lists of $\mathrm{m}^{6} \mathrm{~A}$ methylation correlated genes and upmethylated genes simultaneously, Metascape can identify commonly-enriched and selectively-enriched pathways from two levels, which enable a comprehensive assessment of the molecular features of the biological process. For Multi-list Enrichment Analysis, Metascape first applies enrichment analysis to each gene list individually and identifies terms that are statistically enriched. All gene lists are then combined into one new list, and enrichment analysis is conducted on this combined list. Distinguishing it from many existing portals, Metascape automatically clusters enriched terms into non-redundant groups. Briefly, pairwise similarities between any two enriched terms are computed based on a Kappa-test score. The similarity matrix is then hierarchically clustered 
and a 0.3 similarity threshold is applied to trim the resultant tree into separate clusters. We selected the top 20 clusters and chose the most significant (lowest P-value) term within each of them to represent it.

\subsection{Protein-protein interaction (PPI) network construction of $\mathrm{m}^{6} \mathrm{~A}$ methylation correlated genes}

To find out the functional associations among the $\mathrm{m}^{6} \mathrm{~A}$ methylation correlated genes, we used the online Search Tool for the Retrieval of Interacting Genes (STRING database; http://string-db.org/) to construct a PPI network based on uniquely comprehensive coverage and predictive function of genome-wide data(28). A stringent threshold of a combined score of $>0.7$ was used to construct the PPI network and Cytoscape software was used to visualize and analyze the biological networks. Plugin Molecular Complex Detection (MCODE) was applied to identify significant clusters with strong protein-protein linkages with default parameters.

\subsection{Estimation of immunocyte infiltration}

The CIBERSORT algorithm (https://cibersort.stanford.edu/) was applied to estimate the abundance of infiltrated immune cell subtypes in the lung tissues of 18 samples, based on a deconvolution algorithm in the R software(29). The perm parameter was set as 1000 . The 18 samples were grouped to 'High $\mathrm{m}^{6} \mathrm{~A}$ methylation' and 'Low $\mathrm{m}^{6} \mathrm{~A}$ methylation' by the median value of their $\mathrm{m}^{6} \mathrm{~A}$ methylation score. Comparisons between two groups were tested by the Wilcox rank test. P-value $<0.05$ was considered significant.

\subsection{Statistical analysis}

Briefly, Paired-end reads were harvested from Illumina HiSeq 4000 sequencer, and were quality controlled by Q30. After 3' adaptor-trimming and low quality reads removing by cutadapt software (v1.9.3). First, clean reads of all libraries were aligned to the reference genome (UCSC RN5) by Hisat2 software (v2.0.4). Methylated sites on RNAs (peaks) were identified by MACS software. Differentially methylated sites with a fold change cutoff of $\geq 2$ and false discovery rate cutoff of $\leq 0.0001$ were identified with the diffReps differential analysis package. These peaks identified by both softwares overlapping with exons of mRNA were figured out and choosed by home-made scripts.

\section{Results}

\subsection{Construction of rats PAH model}

The pulmonary artery velocity diagram appeared as a dagger in PAH group, and PAAT consumedly shortened compared to control group $(20.33 \pm 2.62$ vs $29.50 \pm 0.50 \mathrm{~ms}, p<0.0001)$. Then we observed ventricular septum significantly shift to the left, simultaneously, right atrium and right ventricle enlarged compared to control group ( $4.97 \pm 0.30$ vs $3.42 \pm 0.09 \mathrm{~mm}, \mathrm{p}<0.0001)$. The range of TAPSE was greatly reduced in PAH (1.33 \pm 0.05 vs $1.58 \pm 0.04 \mathrm{~mm}, \mathrm{p}<0.0001)$ (Fig. 1.A). Four weeks after the MCT injection, the right ventricular systolic pressure (RVSP) of the PAH group was elevated to $49.87 \pm 1.17 \mathrm{mmHg}$ 
compared with $24.84 \pm 0.34 \mathrm{mmHg}$ in the control $(p<0.001)$, and at the same time, we found that RV/ $(\mathrm{LV}+\mathrm{S})$ increased in PAH group $(0.51 \pm 0.03$ vs $0.25 \pm 0.04 \mathrm{~g}, \mathrm{p}<0.0001)$ (Fig. 1.B). The results of HE staining showed that the pulmonary artery smooth muscle was significantly thickened in PAH group compared with control group $(71.13 \pm 4.02 \%$ vs. $17.95 \pm 1.35 \%, p<0.001)$ (Fig. 1.C). These results indicate that the rat model of PAH has been successfully established.

[Insert Fig. 1]

\subsection{Methylations profile of rat lung tissue}

MeRIP-Seq analysis identified 922 non-overlapping $\mathrm{m}^{6} \mathrm{~A}$ sites in control group and 9059 non-overlapping $\mathrm{m}^{6} \mathrm{~A}$ sites in PAH group, while $18655 \mathrm{~m}^{6} \mathrm{~A}$ sites were overlapped in two groups (Fig. 2.A). Next, differentially methylated $\mathrm{m}^{6} \mathrm{~A}$ sites (DMMSs) were identified by diffReps with the following default screening criteria: $p$-value $\leq 0.0001$ and fold change $\geq 2$ between the groups. We selected 3298 DMMSs in the two groups. A total of $777 \mathrm{~m}^{6} \mathrm{~A}$ sites exhibited up-methylation, and 2521 exhibited downmethylation. (Fig. 2.B). These results demonstrated an increased $\mathrm{m}^{6} \mathrm{~A}$ modification process during PAH and the 2521 up-methylated $\mathrm{m}^{6} \mathrm{~A}$ sites representing 1261 unique genes were selected for further analysis.

[Insert Fig. 2]

\subsection{Construction of weighted gene co-expression network and identification of key modules}

The top 2000 genes in 18 samples of PAH lung tissue were used to construct the co-expression network. $\beta$ values of 5 were confirmed to obtain the approximate scale-free topology with a scale-free topology fit index $>0.85$ and the lowest power (Fig. 3.A). Next, the method of dynamic tree cutting was employed to produce co-expression modules, which led to the identification of 11 modules (Fig. 3.B). We then calculated and plotted the relation of each module with the $\mathrm{m}^{6} \mathrm{~A}$ methylation score. Among these modules, the yellow module depicting the highest correlation (module-trait weighted correlation $=0.46, p$ $=0.055$ ) with $\mathrm{m}^{6} \mathrm{~A}$ methylation score (Figrue 4). Within the yellow module, a total of 87 genes were positively correlative with $\mathrm{m}^{6} \mathrm{~A}$ methylation score and were made subjected to further analysis.

[Insert Fig. 3 and Fig. 4]

\subsection{Multi-list pathway enrichment analysis and visualization}

We conducted a multi-list pathway enrichment analysis in Metascape by inputting the list of $87 \mathrm{~m}^{6} \mathrm{~A}$ methylation correlated genes identified from WGCNA and $1261 \mathrm{~m}^{6} \mathrm{~A}$ upmethylated genes identified from MeRIP-SEq. As Fig. 5 shown, both $\mathrm{m}^{6} \mathrm{~A}$ correlated genes and upmethylated genes are involved in immune response-related pathways such as 'cytokine-mediated signaling pathway', 'leukocyte migration', and 'activation of immune response'. Besides, the upmethylated genes also participate in some biological processes already known to be associate with PAH such as 'ECM organization' and 'supermolecular fiber 
organization'. In sum, the results demonstrated that $\mathrm{m}^{6} \mathrm{~A}$ methylation may play an important role in PAH pathogenesis, especially in mediating immune response during PAH.

[Insert Fig. 5]

\subsection{Identification of hub genes in the yellow module through $\mathrm{PPI}$ analysis}

To identifiy hub genes, we submitted the gene list of $87 \mathrm{~m}^{6} \mathrm{~A}$ correlated genes in the yellow module to STRING for protein-protein interaction analysis, and the cut-off confidence interval was set to 0.7. After MCODE algorithm, the most significant cluster containing 10 hub genes was identified and enrichment analysis shows that genes in this cluster were mainly involved in 'Chemokine receptors bind chemokines' pathway (Fig. 6). By comparing the results of MeRIP-Seq, CCR5 and CXCL9 were found to be significantly upmethylated with a fold change of 2.55 and 6.93 , respectively.

[Insert Fig. 6]

\subsection{Correlation analysis between $\mathrm{m}^{6} \mathrm{~A}$ methylation and immunocyte proportion}

We have concluded that $\mathrm{m}^{6} \mathrm{~A}$ methylation can mediate immune response during $\mathrm{PAH}$ process. We then aimed to investigate how it regulates immune cell infiltration. After the Wilcox rank test, monocyte and M1 macrophage were detected differentially infiltrated between the high methylation group and low methylation group ( $p=0.03$, and $p=0.001$, respectively) (Fig. 7). Figure 8 displayed the scale histogram of immune cell fraction among 18 samples. These results indicated that during $\mathrm{PAH}$, the $\mathrm{m}^{6} \mathrm{~A}$ methylation can drive monocyte to form M1 macrophage, which plays vital roles in the immune response.

[Insert Fig. 7 and Fig. 8]

\section{Discussion}

$\mathrm{m}^{6} \mathrm{~A}$ is the most prevalent modification in the mRNA of many eukaryotic species. There is growing evidence that $\mathrm{m}^{6} \mathrm{~A}$ dysregulation has a profound impact on the pathogenesis of various human diseases(30-33). Su et al. firstly identified the transcriptome-wide map of $m^{6} A$ circRNAs in hypoxic PAH rat model and confirmed that $\mathrm{m}^{6} \mathrm{~A}$ methylation can affect the circRNA-miRNA-mRNA network(34). However, the mechanism of mRNA $m^{6} A$ methylation modification to regulate IPAH has not been reported. In this study, we took advantage of MeRIP-seq to map the transcriptomic landscape of PAH rat lung tissue and thus quantitatively compared transcriptome-wide changes between $\mathrm{PAH}$ and control groups. The results found that the $\mathrm{m}^{6} \mathrm{~A}$ level was significantly up-regulated in MCT induced PAH lung tissue, which highlighted the role of $\mathrm{m}^{6} \mathrm{~A}$ methylation in the pathogenesis of PAH. 
WGCNA is a method for constructing gene co-expression networks based on gene expression data. We applied this method to identified key modules associated with the estimated $\mathrm{m}^{6} \mathrm{~A}$ methylation score. Through multi-list enrichment analysis, the $87 \mathrm{~m}^{6} \mathrm{~A}$ methylation correlated genes identified from WGCNA and $1261 \mathrm{~m}^{6} \mathrm{~A}$ up-methylated genes identified from MeRIP-seq analysis are both significantly involved in immune response-related pathways such as 'cytokine-mediated signaling pathway', 'leukocyte migration', and 'activation of immune response'. These results highly suggested the immunoregulatory role of $\mathrm{m}^{6} \mathrm{~A}$ methylation during $\mathrm{PAH}$.

Through PPI analysis of the $87 \mathrm{~m}^{6} \mathrm{~A}$ methylation correlated genes, a cluster containing 10 hub genes were identified, including CXCL9, CXCL10, CXCR3, PMCH, CXCL11, CXCR6, CCR7, CCL5, CCR5, and HCAR3. Among them, CCR5 and CXCL9 were significantly up-methylated. CCR5 is activated on stimulation by the CCR5 ligands CCL3 (macrophage inflammatory protein-1a), CCL4 (macrophage inflammatory protein-1 $\beta$ ), and CCL5 (RANTES) and is strongly expressed on the principal cell types implicated in PAH progression, including endothelial cells, SMCs, T cells, and macrophages(35-38). Amsellem et al. studied the effect of CCR5 receptor antagonists on PASMC and inflammatory response in PAH mouse model and found that the activation of the CCL5-CCR5 axis directly leads to PASMC proliferation and macrophage recruitment(39). CXCL9, a chemokine, is a T-cell chemoattractant that is induced by IFN- $\gamma$. CXCL9 is closely related to two other CXC chemokines called CXCL10 and CXCL11, whose genes are located near the gene for CXCL9 on human chromosome 4(40,41). CXCL9, CXCL10, and CXCL11 are commonly produced by local cells in inflammatory lesions and can attract Th1 cells(42). A high level of CXCL9 in peripheral liquids can be considered as a marker of host immune response, especially of that involving $\operatorname{Th} 1$ cells $(43,44)$.

By applying the CYBERSORT algorithm, we found that the $\mathrm{m}^{6} \mathrm{~A}$ methylation can drive monocyte to form M1 macrophage. Circulating monocytes migrate into the majority of tissues in the body, where they differentiate into functionally distinct mature macrophages $(45,46)$. Macrophages undergo classical M1 activation induced by IFN- $\gamma$, which mediates Th1 cell-type activation of macrophages(47). The activated macrophage then causes vasoconstriction, increases vascular permeability, and induce PASMCs proliferation(48). Our research sheld light on the regulatory role of $\mathrm{m}^{6} \mathrm{~A}$ methylation in macrophage activation during PAH. As we discussed above, The hub $10 \mathrm{~m}^{6} \mathrm{~A}$ methylation correlated genes,especially CCR5 and CXCL9ロmay mediate $\mathrm{m}^{6} \mathrm{~A}$ methylation caused M1 macrophage activation.

\section{Conclusions}

In conclusion, our research revealed that $\mathrm{m}^{6} \mathrm{~A}$ methylation modification may play important roles in mediating immune response during PAH. It also caused activation of M1macrophage, which may be mediated by CCR5 and CXCL9. These results will help us to better understand the mechanisms of PAH, and provide candidate therapeutic targets.

\section{Abbreviations}




\section{DMMSs}

differentially methylated m6A sites

$\mathrm{m} 6 \mathrm{~A}$

N6-methyladenosine

MCT

monocrotaline

MeRIP-Seq

Methylated RNA immunoprecipitation sequencing

$\mathrm{PAH}$

pulmonary arterial hypertension

PAAT

pulmonary artery blood flow acceleration time

PPI

protein-protein interaction

$\mathrm{RV} /(\mathrm{LV}+\mathrm{S})$

right ventricle to left ventricle plus ventricular septum

RVSP

right ventricular systolic pressure

TAPSE

tricuspid annular plane systolic excursion

WGCNA

weighted gene co-expression network analysis

\section{Declarations}

\section{Ethics approval and consent to participate}

The study was approved by the Research Ethics Committee of Second Xiangya Hospital of Central South University. The research was conducted according to all ethical standards.

\section{Consent for publication}

Consent to publish was obtained from all authors.

\section{Availability of data and materials}

All data in our study are available upon request.

\section{Competing interests}

The authors declare that they have no competing interests.

\section{Funding}


This project was supported by grants from the National Natural Science Foundation of China (No. 81770337). This project was supported by grants from the Hunan Provincial Natural Science Youth Foundation of China (No. 2019JJ50881). Hunan Provincial Health Commission Scientific Research Project (NO. 20201302). Hunan Province Traditional Chinese Medicine Research Program Project (No. 201914).

\section{Author's contributions}

QML and YBX conceived and designed this study; NL and YZL performed experiments, analyzed and interpreted data, and wrote the manuscript; $\mathrm{YHZ}$ and $\mathrm{HT}$ designed the microarray and analyzed the microarray data; $\mathrm{BL}$ and ZHY collected clinical samples, interpreted data and clinical information; QML improved and revised the manuscript; NL and YZL analyzed and interpreted data, supervised experiments, and wrote the manuscript. All authors read and approved the final manuscript.

\section{Ackonwledgements}

We are grateful to CloudSeq Biotech Inc. for scientific support.

\section{References}

1. Thenappan T, Ormiston ML, Ryan JJ, Archer SL. Pulmonary arterial hypertension: pathogenesis and clinical management. BMJ. 2018;360:j5492.

2. Simonneau G, Gatzoulis MA, Adatia I, Celermajer D, Denton C, Ghofrani A, et al. Updated clinical classification of pulmonary hypertension. J Am Coll Cardiol. 2013;62(25 Suppl):D34-41.

3. Jacobs W, van de Veerdonk MC, Trip P, de Man F, Heymans MW, Marcus JT, et al. The right ventricle explains sex differences in survival in idiopathic pulmonary arterial hypertension. Chest. 2014;145(6):1230-6.

4. Hatton N, Ryan JJ. Sex differences in response to pulmonary arterial hypertension therapy: is what's good for the goose, good for the gander? Chest. 2014;145(6):1184-6.

5. Liu N, Parry S, Xiao Y, Zhou S, Liu Q. Molecular targets of the Warburg effect and inflammatory cytokines in the pathogenesis of pulmonary artery hypertension. Clin Chim Acta. 2017;466:98-104.

6. Cantara WA, Crain PF, Rozenski J, McCloskey JA, Harris KA, Zhang X, et al. The RNA Modification Database, RNAMDB: 2011 update. Nucleic Acids Res. 2011;39(Database issue):D195-201.

7. Liu N, Pan T. N6-methyladenosine-encoded epitranscriptomics. Nat Struct Mol Biol. 2016;23(2):98102.

8. Li S, Mason CE. The pivotal regulatory landscape of RNA modifications. Annu Rev Genom Hum Genet. 2014;15:127-50.

9. Liu J, Yue Y, Han D, Wang X, Fu Y, Zhang L, et al. A METTL3-METTL14 complex mediates mammalian nuclear RNA N6-adenosine methylation. Nature chemical biology. 2014;10(2):93-5. 
10. Jia G, Fu Y, Zhao X, Dai Q, Zheng G, Yang Y, et al. N6-methyladenosine in nuclear RNA is a major substrate of the obesity-associated FTO. Nature chemical biology. 2011;7(12):885-7.

11. Tang $\mathrm{C}$, Klukovich R, Peng H, Wang Z, Yu T, Zhang Y, et al. ALKBH5-dependent m6A demethylation controls splicing and stability of long 3'-UTR mRNAs in male germ cells. Proc Natl Acad Sci USA. 2018;115(2):E325-e33.

12. Xu C, Wang X, Liu K, Roundtree IA, Tempel W, Li Y, et al. Structural basis for selective binding of m6A RNA by the YTHDC1 YTH domain. Nature chemical biology. 2014;10(11):927-9.

13. Li F, Zhao D, Wu J, Shi Y. Structure of the YTH domain of human YTHDF2 in complex with an m(6)A mononucleotide reveals an aromatic cage for m(6)A recognition. Cell Res. 2014;24(12):1490-2.

14. Liu N, Zhou KI, Parisien M, Dai Q, Diatchenko L, Pan T. N6-methyladenosine alters RNA structure to regulate binding of a low-complexity protein. Nucleic Acids Res. 2017;45(10):6051-63.

15. Pendleton KE, Chen B, Liu K, Hunter OV, Xie Y, Tu BP, et al. The U6 snRNA m(6)A Methyltransferase METTL16 Regulates SAM Synthetase Intron Retention. Cell. 2017;169(5):824 - 35.e14.

16. Yang $X$, Yang Y, Sun BF, Chen YS, Xu JW, Lai WY, et al. 5-methylcytosine promotes mRNA export NSUN2 as the methyltransferase and ALYREF as an m(5)C reader. Cell Res. 2017;27(5):606-25.

17. Zheng G, Dahl JA, Niu Y, Fedorcsak P, Huang CM, Li CJ, et al. ALKBH5 is a mammalian RNA demethylase that impacts RNA metabolism and mouse fertility. Molecular cell. 2013;49(1):18-29.

18. Zhao BS, Wang X, Beadell AV, Lu Z, Shi H, Kuuspalu A, et al. m(6)A-dependent maternal mRNA clearance facilitates zebrafish maternal-to-zygotic transition. Nature. 2017;542(7642):475-8.

19. Wang X, Lu Z, Gomez A, Hon GC, Yue Y, Han D, et al. N6-methyladenosine-dependent regulation of messenger RNA stability. Nature. 2014;505(7481):117-20.

20. Shi H, Wang X, Lu Z, Zhao BS, Ma H, Hsu PJ, et al. YTHDF3 facilitates translation and decay of N(6)methyladenosine-modified RNA. Cell Res. 2017;27(3):315-28.

21. Li A, Chen YS, Ping XL, Yang $X$, Xiao W, Yang Y, et al. Cytoplasmic $m(6) A$ reader YTHDF3 promotes mRNA translation. Cell Res. 2017;27(3):444-7.

22. Rabinovitch $M$, Guignabert $C$, Humbert $M$, Nicolls MR. Inflammation and immunity in the pathogenesis of pulmonary arterial hypertension. Circulation research. 2014;115(1):165-75.

23. Shulman Z, Stern-Ginossar N. The RNA modification N(6)-methyladenosine as a novel regulator of the immune system. Nature immunology. 2020;21(5):501-12.

24. Langfelder $P$, Horvath $S$. WGCNA: an R package for weighted correlation network analysis. BMC Bioinformatics. 2008;9:559.

25. Y Y, PJ H, YS C. YG Y. Dynamic transcriptomic $m A$ decoration: writers, erasers, readers and functions in RNA metabolism. Cell research. 2018;28(6):616-24.

26. S H, R C, J G. GSVA: gene set variation analysis for microarray and RNA-seq data. BMC bioinformatics. 2013;14:7.

27. Zhou Y, Zhou B, Pache L, Chang M, Khodabakhshi AH, Tanaseichuk O, et al. Metascape provides a biologist-oriented resource for the analysis of systems-level datasets. Nat Commun. 
2019;10(1):1523.

28. Szklarczyk D, Morris JH, Cook H, Kuhn M, Wyder S, Simonovic M, et al. The STRING database in 2017: quality-controlled protein-protein association networks, made broadly accessible. Nucleic Acids Res. 2017;45(D1):D362-D8.

29. Newman AM, Liu CL, Green MR, Gentles AJ, Feng W, Xu Y, et al. Robust enumeration of cell subsets from tissue expression profiles. Nat Methods. 2015;12(5):453-7.

30. Yoon KJ, Ringeling FR, Vissers C, Jacob F, Pokrass M, Jimenez-Cyrus D, et al. Temporal Control of Mammalian Cortical Neurogenesis by m(6)A Methylation. Cell. 2017;171(4):877 - 89.e17.

31. Mathiyalagan P, Adamiak M, Mayourian J, Sassi Y, Liang Y, Agarwal N, et al. FTO-Dependent N(6)Methyladenosine Regulates Cardiac Function During Remodeling and Repair. Circulation. 2019;139(4):518-32.

32. Dorn LE, Lasman L, Chen J, Xu X, Hund TJ, Medvedovic M, et al. The N(6)-Methyladenosine mRNA Methylase METTL3 Controls Cardiac Homeostasis and Hypertrophy. Circulation. 2019;139(4):53345.

33. Berulava T, Buchholz E, Elerdashvili V, Pena T, Islam MR, Lbik D, et al. Changes in m6A RNA methylation contribute to heart failure progression by modulating translation. Eur J Heart Fail. 2020;22(1):54-66.

34. Su H, Wang G, Wu L, Ma X, Ying K, Zhang R. Transcriptome-wide map of m(6)A circRNAs identified in a rat model of hypoxia mediated pulmonary hypertension. BMC Genomics. 2020;21(1):39.

35. Schober A. Chemokines in vascular dysfunction and remodeling. Arteriosclerosis, thrombosis, and vascular biology. 2008;28(11):1950-9.

36. Charo IF, Ransohoff RM. The many roles of chemokines and chemokine receptors in inflammation. $\mathrm{N}$ Engl J Med. 2006;354(6):610-21.

37. Schecter AD, Calderon TM, Berman AB, McManus CM, Fallon JT, Rossikhina M, et al. Human vascular smooth muscle cells possess functional CCR5. J Biol Chem. 2000;275(8):5466-71.

38. Ishida Y, Kimura A, Kuninaka Y, Inui M, Matsushima K, Mukaida N, et al. Pivotal role of the CCL5/CCR5 interaction for recruitment of endothelial progenitor cells in mouse wound healing. J Clin Investig. 2012;122(2):711-21.

39. Amsellem V, Lipskaia L, Abid S, Poupel L, Houssaini A, Quarck R, et al. CCR5 as a treatment target in pulmonary arterial hypertension. Circulation. 2014;130(11):880-91.

40. Tokunaga R, Zhang W, Naseem M, Puccini A, Berger MD, Soni S, et al. CXCL9, CXCL10, CXCL11/CXCR3 axis for immune activation - A target for novel cancer therapy. Cancer treatment reviews. 2018;63:40-7.

41. Van Raemdonck K, Van den Steen PE, Liekens S, Van Damme J, Struyf S. CXCR3 ligands in disease and therapy. Cytokine Growth Factor Rev. 2015;26(3):311-27.

42. Smit MJ, Verdijk P, van der Raaij-Helmer EM, Navis M, Hensbergen PJ, Leurs R, et al. CXCR3-mediated chemotaxis of human $\mathrm{T}$ cells is regulated by a $\mathrm{Gi}$ - and phospholipase $\mathrm{C}$-dependent pathway and not 
via activation of MEK/p44/p42 MAPK nor Akt/PI-3 kinase. Blood. 2003;102(6):1959-65.

43. Antonelli A, Ferrari SM, Giuggioli D, Ferrannini E, Ferri C, Fallahi P. Chemokine (C-X-C motif) ligand (CXCL)10 in autoimmune diseases. Autoimmun rev. 2014;13(3):272-80.

44. Liu C, Papewalis C, Domberg J, Scherbaum WA, Schott M. Chemokines and autoimmune thyroid diseases. Hormone and metabolic research $=$ Hormon- und Stoffwechselforschung $=$ Hormones et metabolisme. 2008;40(6):361-8.

45. Chaudhuri A. Regulation of Macrophage Polarization by RON Receptor Tyrosine Kinase Signaling. Frontiers in immunology. 2014;5:546.

46. Schultze JL, Schmieder A, Goerdt S. Macrophage activation in human diseases. Seminars in immunology. 2015;27(4):249-56.

47. Wang $\mathrm{N}$, Liang $\mathrm{H}$, Zen $\mathrm{K}$. Molecular mechanisms that influence the macrophage $\mathrm{m} 1$-m2 polarization balance. Frontiers in immunology. 2014;5:614.

48. Colvin KL, Cripe PJ, Ivy DD, Stenmark KR, Yeager ME. Bronchus-associated lymphoid tissue in pulmonary hypertension produces pathologic autoantibodies. Am J Respir Crit Care Med. 2013;188(9):1126-36.

\section{Figures}


A
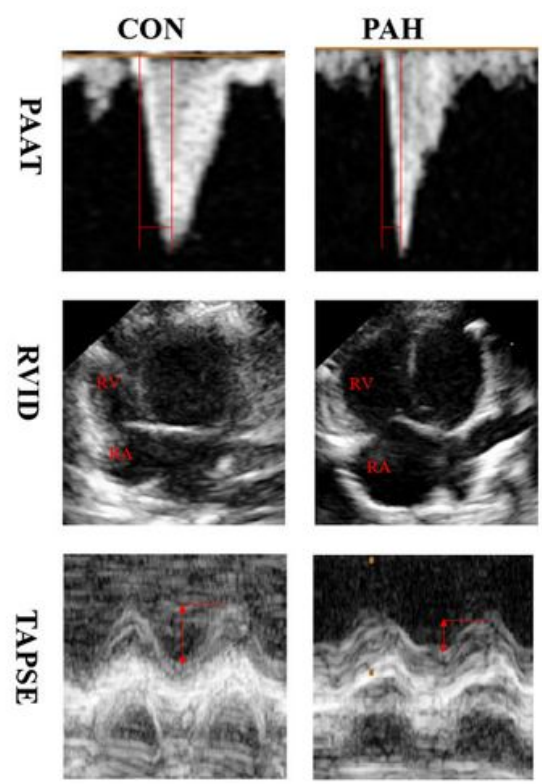

$\mathrm{B}$
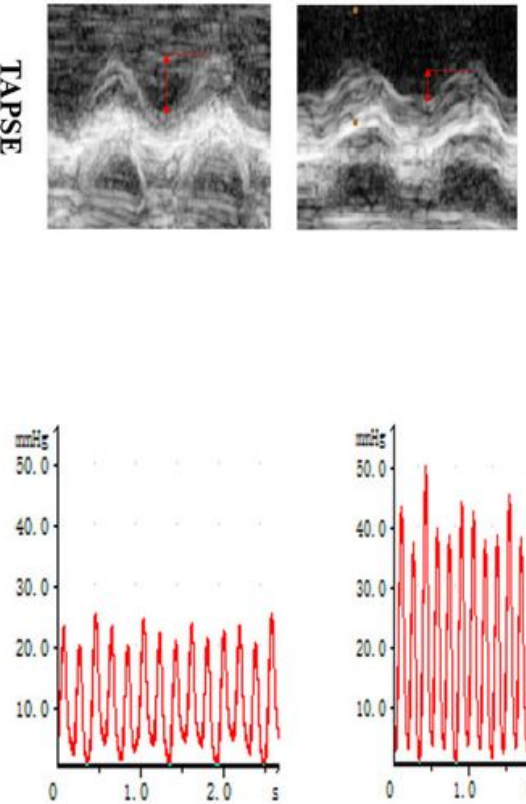
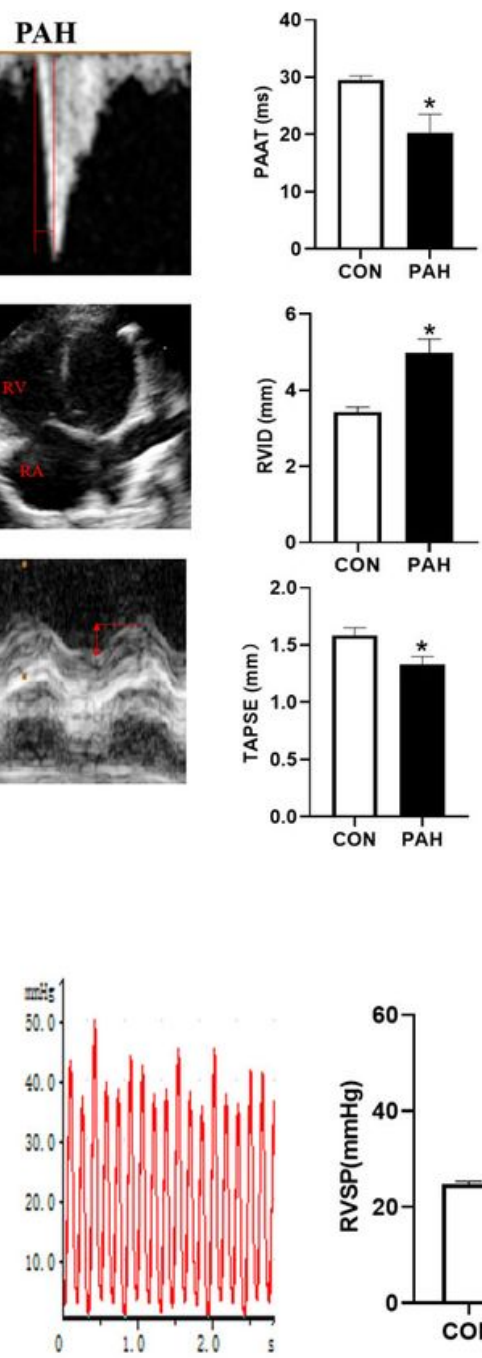

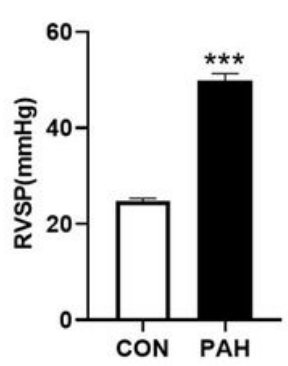

$\mathrm{C}$
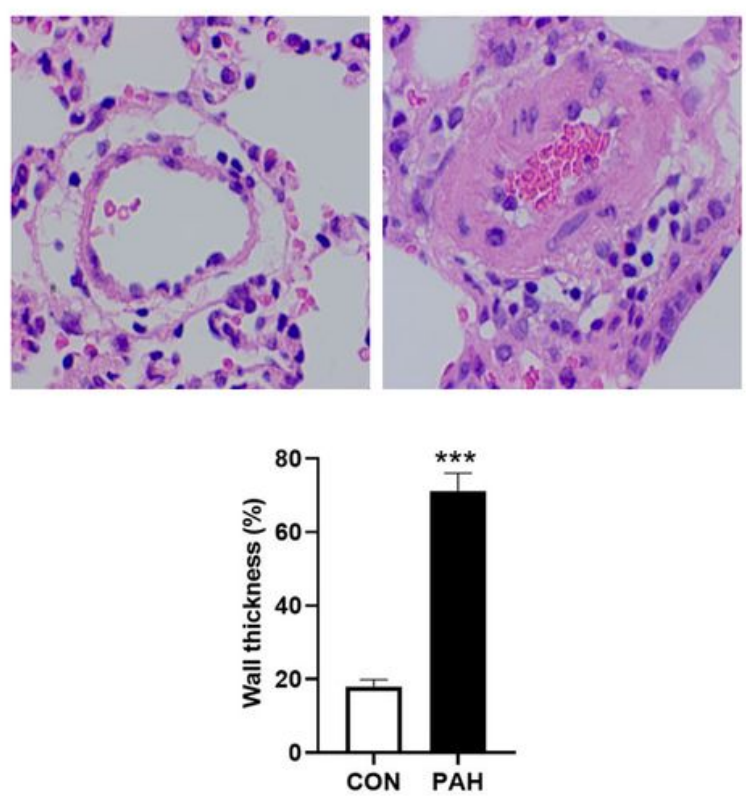

Figure 1

Construction of rats PAH model. (A) Echocardiography data, (B) Hemodynamic data, (C) Pulmonary artery HE staining images of the control group and PAH group were obtained under microscopy. * represents statistical significance. 
A

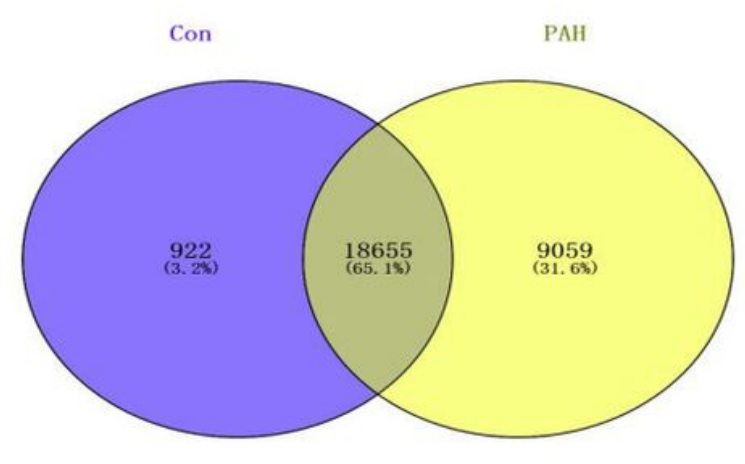

B
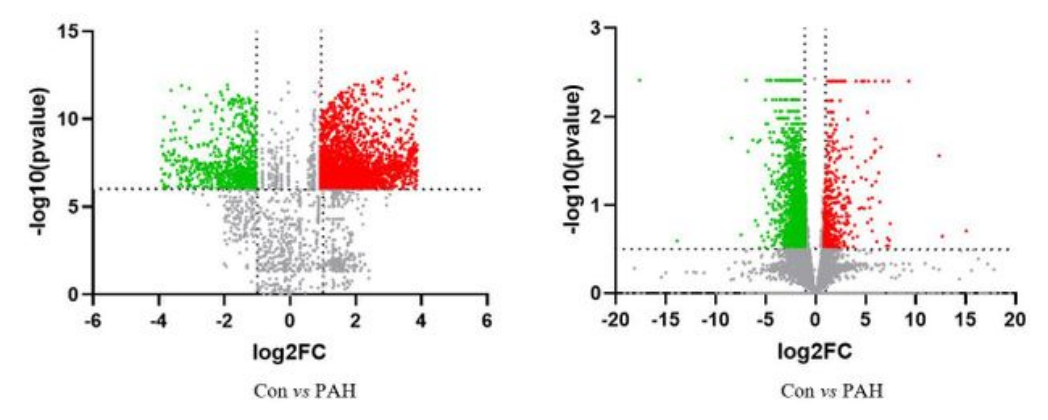

\begin{tabular}{|c|c|c|c|c|}
\hline \multicolumn{5}{|c|}{ Total numbers of differentially methylated $\mathrm{N}$ 'methyladenosine peaks and associated gene } \\
\hline Item & Upmetbylated peak & Upmetbylated geve & Dorrametbylated peak & Dorametbilated geae \\
\hline mRNA & 2521 & 1261 & 777 & 568 \\
\hline
\end{tabular}

\section{Figure 2}

Methylations profile of rat lung tissue. (A) The differential m6A methylation and the expression of mRNA methylation statistical volcano map. Green represents m6A modification and the expression of mRNA methylation is decreased, red is the opposite. (B) Differential m6A enrichment peaks and related genes statistical graph. 
A

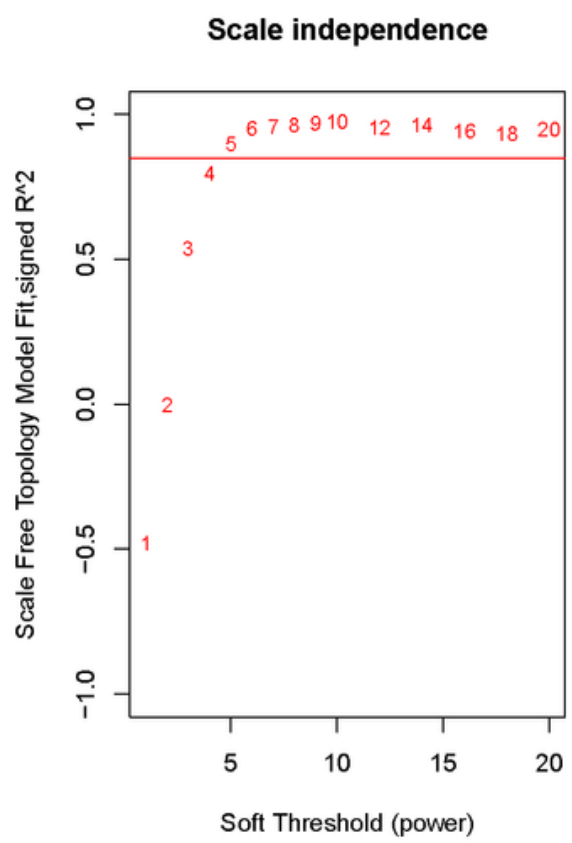

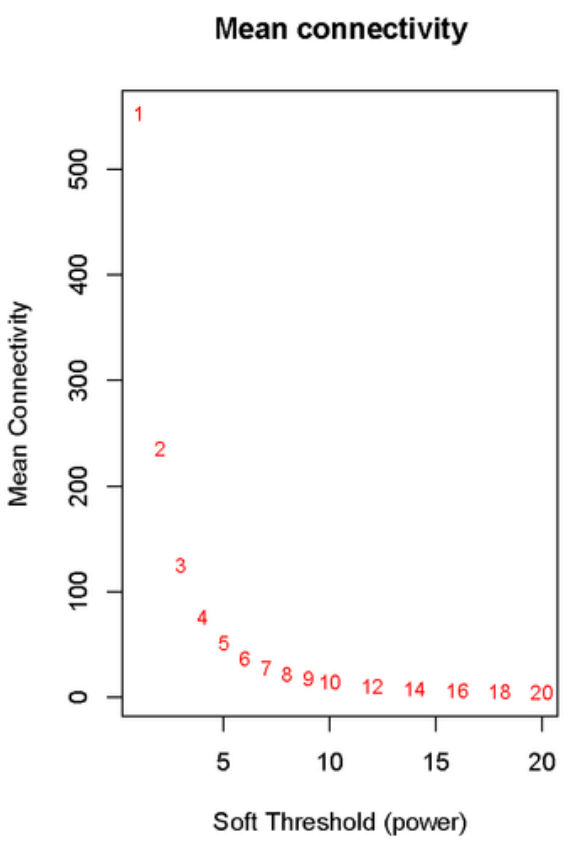

B

\section{Cluster Dendrogram}
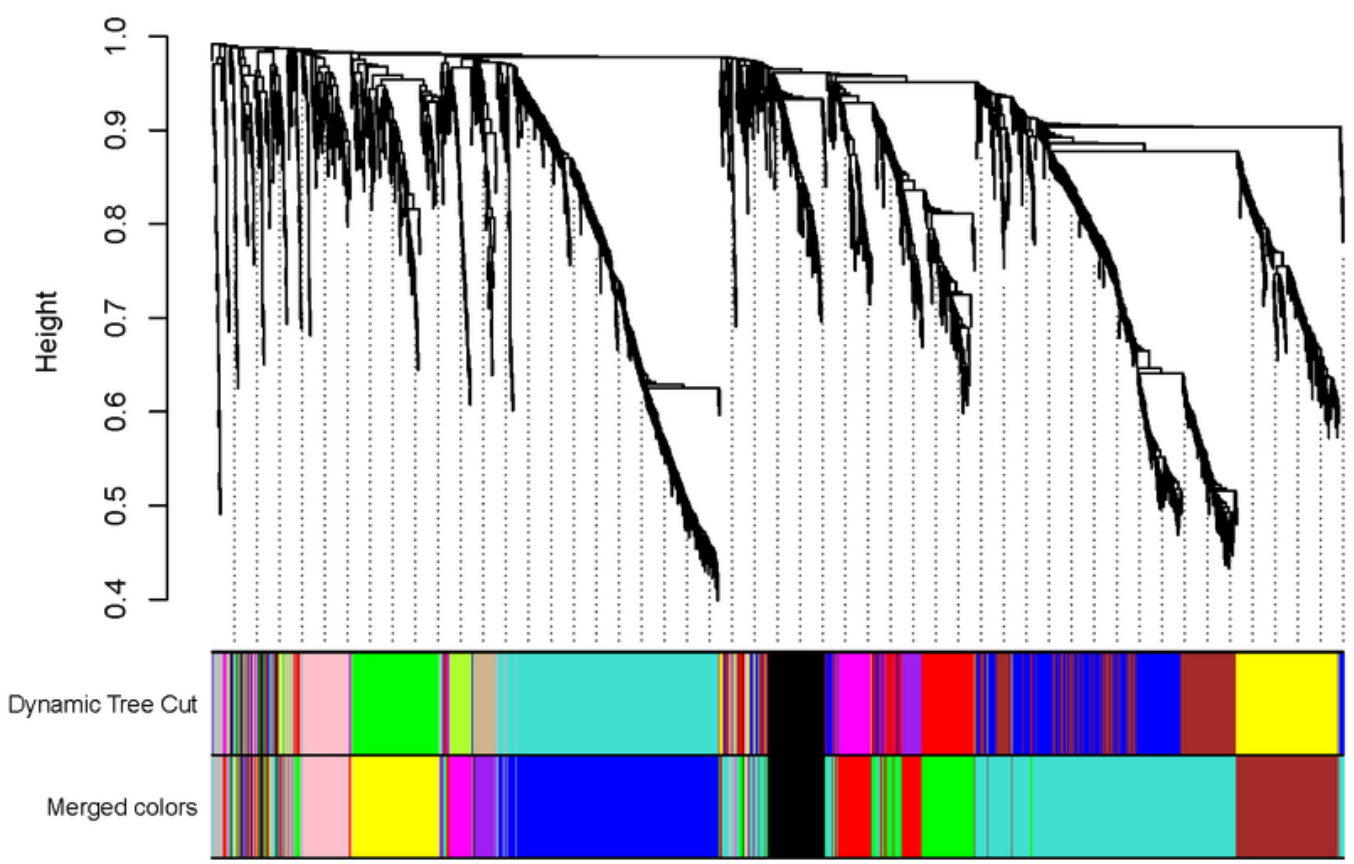

\section{Figure 3}

Methylations profile of rat lung tissue. (A) The differential m6A methylation and the expression of mRNA methylation statistical volcano map. Green represents m6A modification and the expression of mRNA methylation is decreased, red is the opposite. (B) Differential m6A enrichment peaks and related genes statistical graph. 


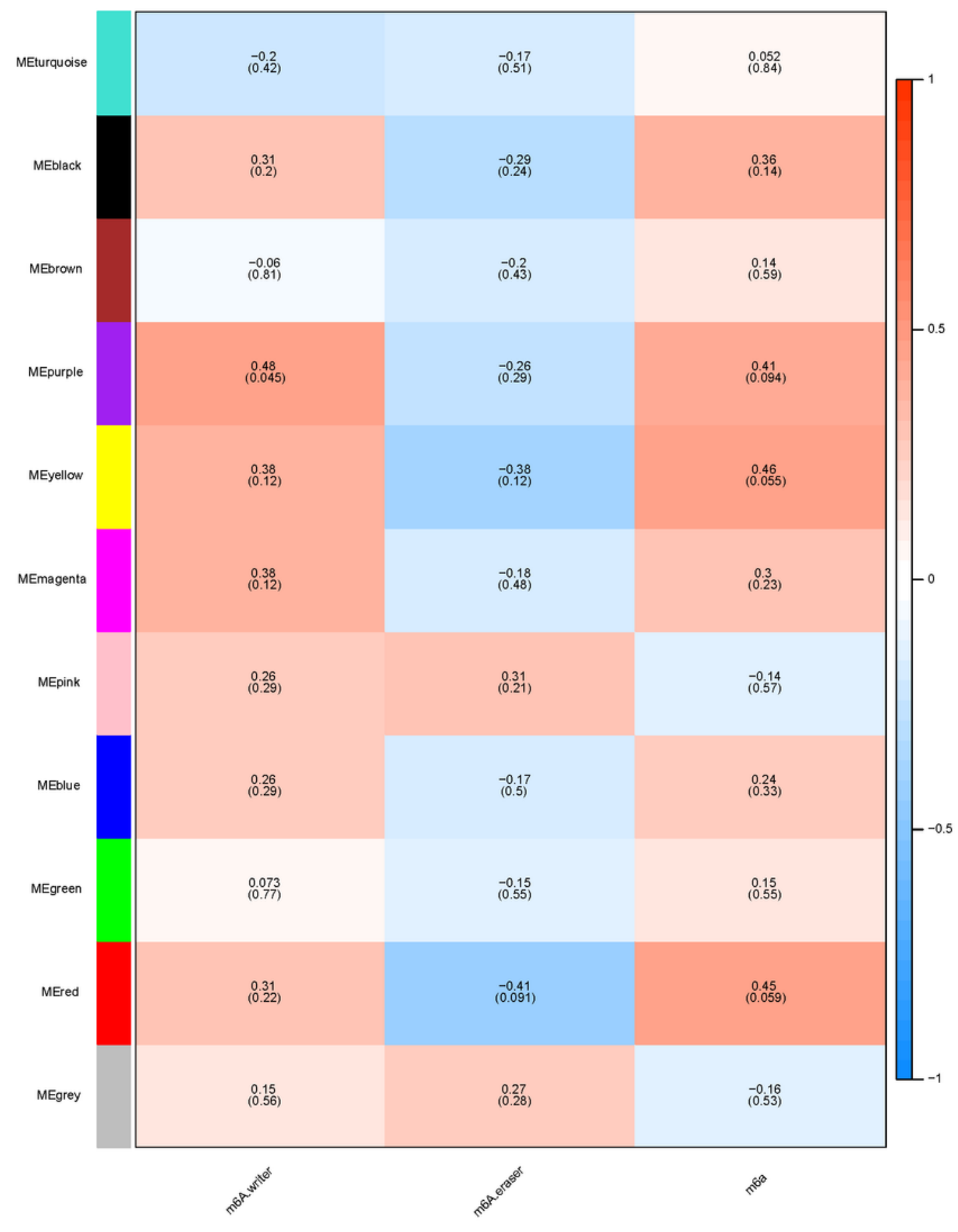

\section{Figure 4}

Heatmap of the correlation between m6A methylation score and module eigengenes. Each row corresponds to a module eigengene, and each column corresponds to a trait. Each cell contains the corresponding correlation (first line) and p-value (second line). The table is color-coded by correlation according to the color legend. 


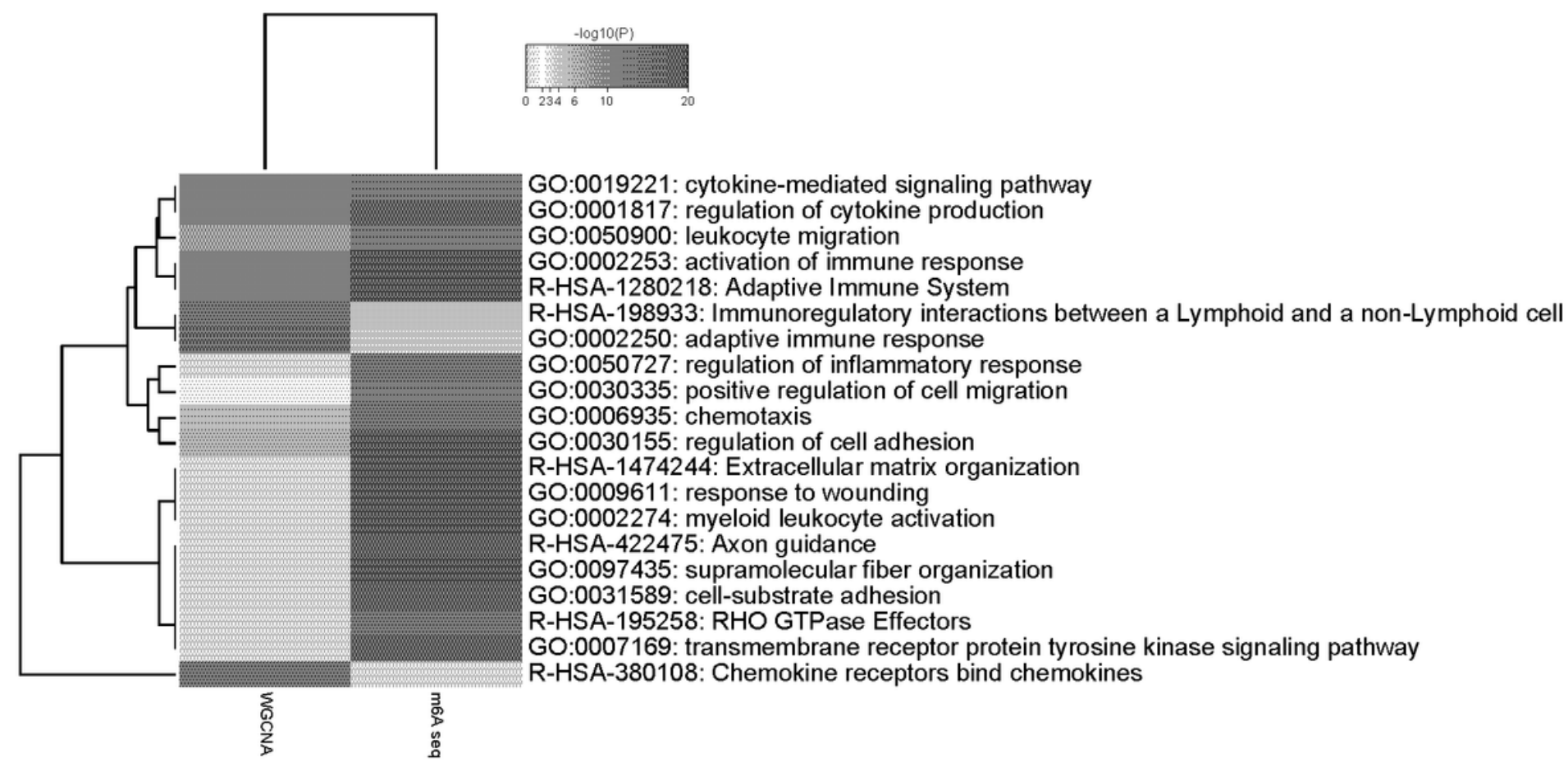

Figure 5

Multi-list enrichment pathway analysis of m6A methylation correlated genes and upmethylated genes. The diagram depicts the top 20 clusters with the smallest $p$-value. 


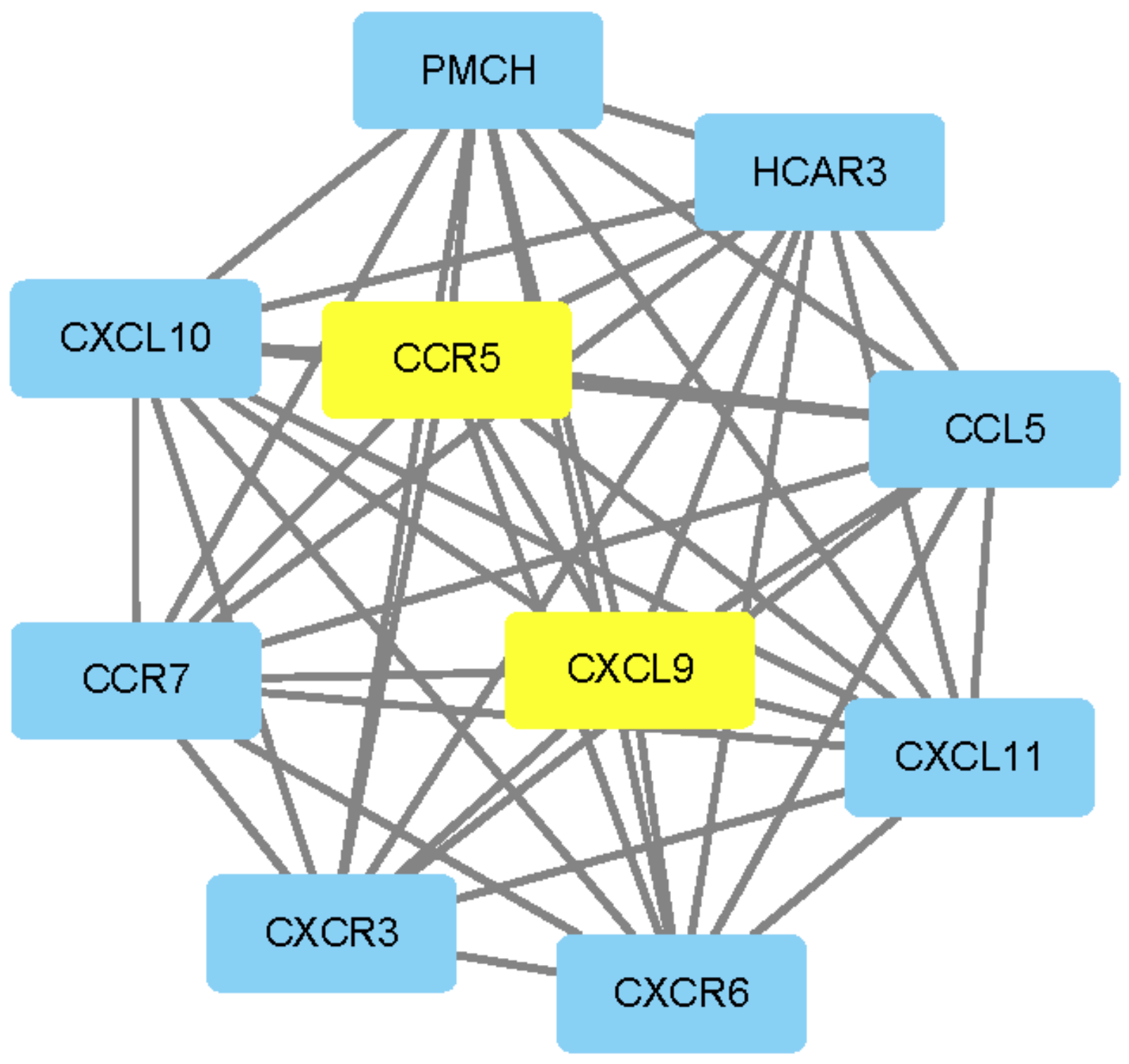

Figure 6

Key cluster of 87 methylation correlated genes identified from PPI analysis. Yellow represents upmethylated genes. 


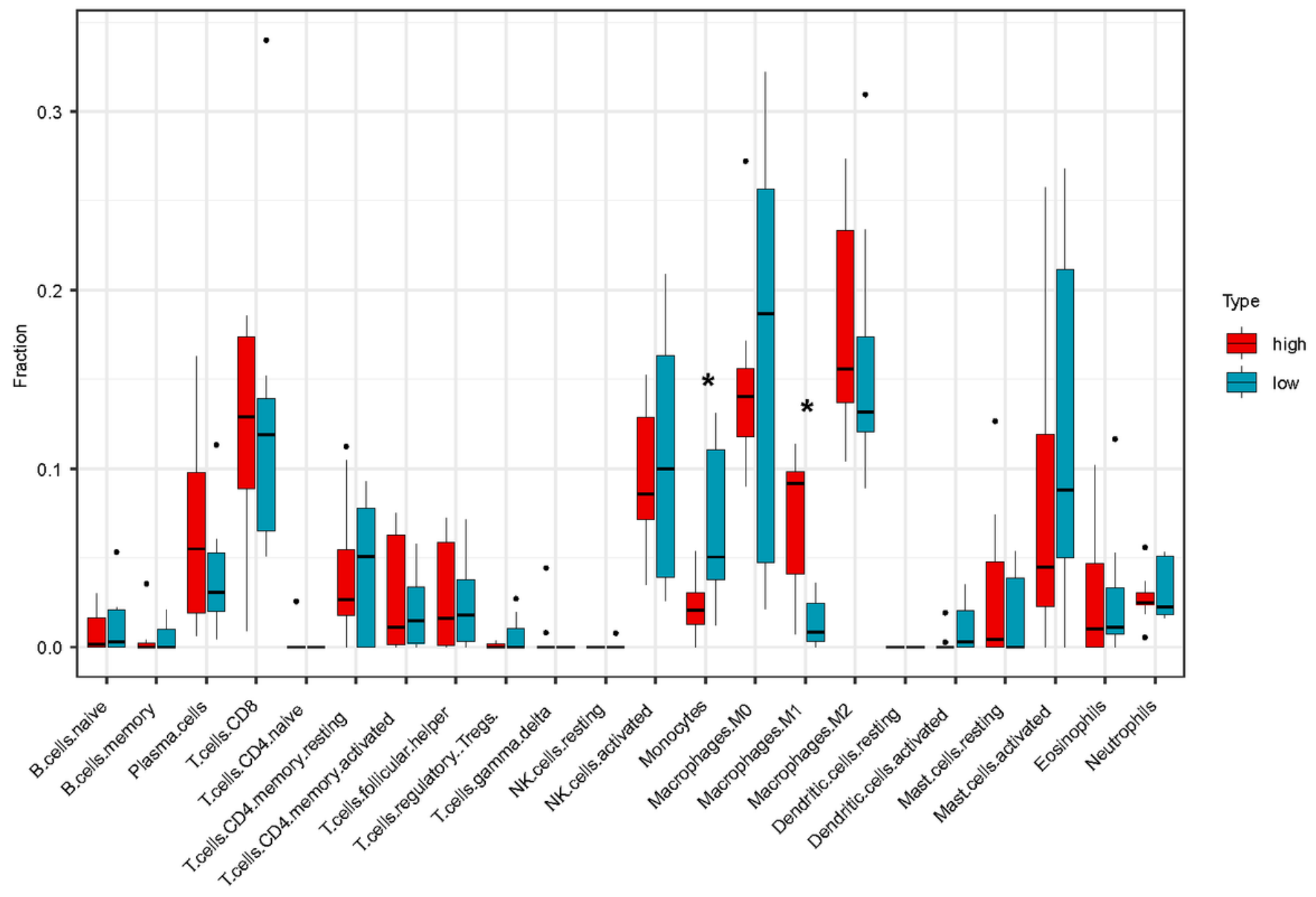

Figure 7

Immune cells composition between high methylation and low methylation group. * represents statistical significance. Black dots represent outliers. 


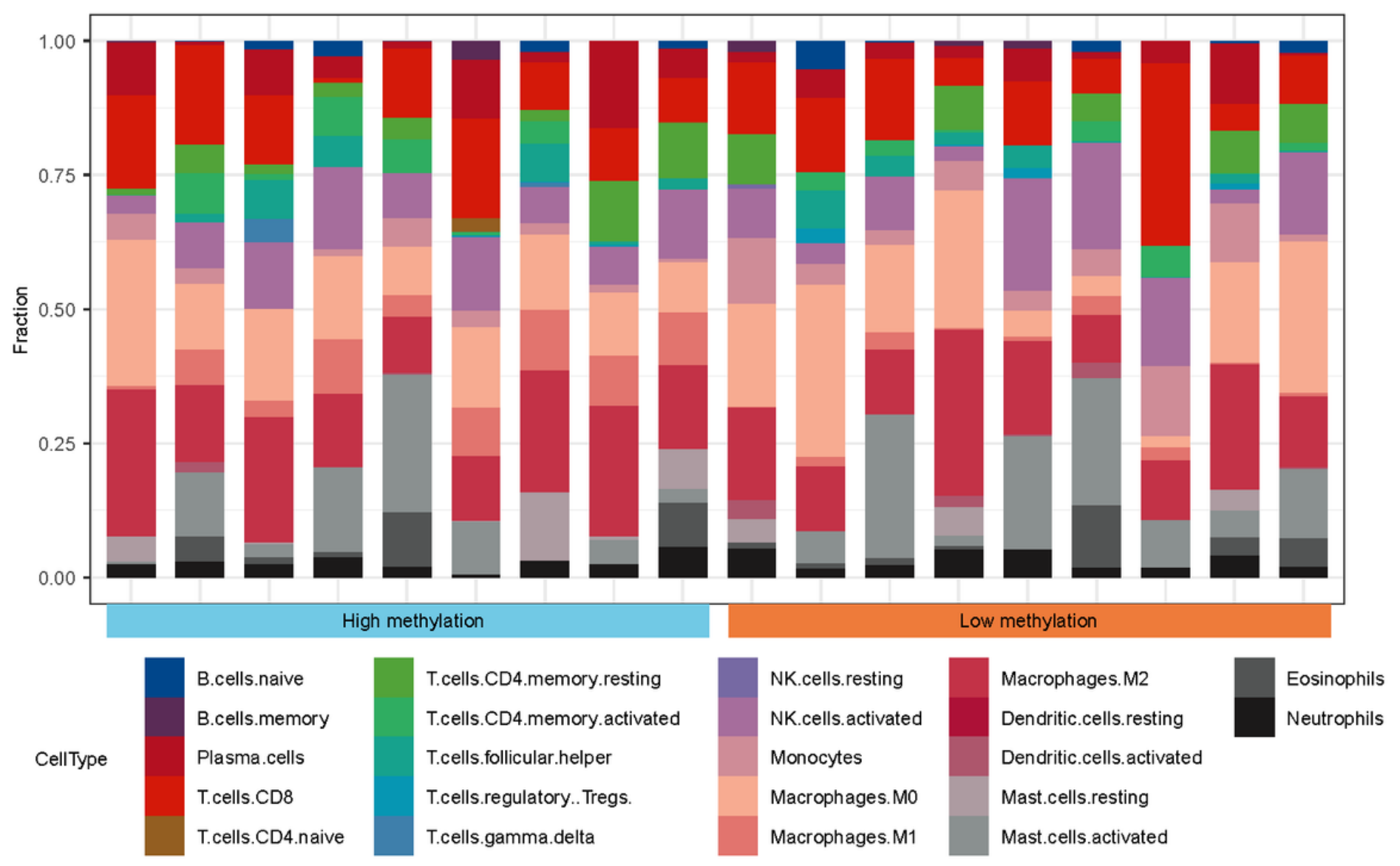

Figure 8

Scale histogram of immune cell fraction among 18 IPAH samples.

\section{Supplementary Files}

This is a list of supplementary files associated with this preprint. Click to download.

- Q30.xlsx

- readstatistics.xlsx

- samplegroups.xlsx 